\title{
Capital social y redes políticas de las asociaciones de inmigrantes en Galicia
}

\author{
Belén Fernández-Suárez \\ Esther Cano-Ruiz \\ Universidade da Coruña \\ belenfernandez@udc.es; esther.cano.ruiz@udc.es
}

\section{Resumen}

Este artículo se centra en analizar la participación política de los extranjeros asentados en Galicia a través del tejido asociativo inmigrante. Se considera que la participación social en entidades creadas por inmigrantes es sinónimo de integración social y favorece la participación política. Esta participación política se divide en dos componentes: el derecho de acceso a la toma de decisiones y la participación efectiva, entendida como los contactos, las prácticas asociativas y las relaciones con actores sociales y políticos.

Partimos de una metodología mixta que combina la explotación de datos de un cuestionario con el análisis de entrevistas cualitativas. A partir del Cuestionario a Asociaciones de Inmigrantes en Galicia, obtenemos datos cuantitativos de 32 asociaciones de inmigrantes sobre las actividades y sus relaciones de tipo institucional y político. Las 43 entrevistas semiestructuradas a líderes asociativos, representantes institucionales y entidades del tercer sector nos posibilitarán que profundicemos en los discursos que sostienen su actuación y su posición ideológica.

Los principales resultados nos señalan una participación política de las entidades de extranjeros circunscrita al ámbito municipal. La existencia de mecanismos formales permite su articulación como actores sociales pero una escasa incidencia política, lo que puede vislumbrarse en un bajo contacto con entidades políticas y sociales. Cuando este contacto se produce, está más dirigido a solventar necesidades derivadas de la vulnerabilidad social que demandas típicas de un proceso de asentamiento consolidado.

Palabras clave: asociacionismo migrante; capital social; participación política; tercer sector; actor político 


\section{Abstract. Social capital and political networks of migrant associations in Galicia}

This article examines foreigners' political participation in Galicia through migrant associations. It considers that social participation in organizations promoted by migrants implies social integration and favors political involvement. This political participation is divided into two elements: the right of access to decision making and effective participation, which is understood as contacts, associative practices and relationships with social and political actors. A mixed methodology is used combining data drawn from a questionnaire and the analysis of qualitative interviews. From the questionnaire administered to immigrant organizations in Galicia, we obtained quantitative data on the activities and institutional and political relations of 32 immigrant associations. The 43 semi-structured interviews of association leaders, institutional representatives and third sector entities allows us to look deeper into the discourses that support their actions and their ideological-political position. The main results point to a political participation of foreigners' organizations circumscribed to the local sphere, as well as to the existence of formal mechanisms that allow their consideration as social actors but with scarce political incidence characterized by little contact with political and social entities. When this occurs, it is more aimed at solving needs derived from social vulnerability rather than the typical demands of a consolidated settlement process.

Keywords: migrant associations; social capital; political involvement; third sector; political actor

\section{Sumario}

1. Introducción: Capital social y redes sociales en el asociacionismo inmigrante 2. Metodología

3. El contexto migratorio gallego

4. Los números de la integración política de las asociaciones de inmigrantes en Galicia
5. Los discursos de asociaciones de inmigrantes y de actores clave sobre la integración política de tejido social migrante

6. Epílogo: ¿De lo global a la estructura local de oportunidades?

Referencias bibliográficas

\section{Introducción: Capital social y redes sociales en el asociacionismo inmigrante}

Esta propuesta busca analizar el papel que desempeñan las relaciones y el capital social a través del tejido asociativo en la integración política de los inmigrantes, para lo cual nos centraremos en estudiar las relaciones de las organizaciones de inmigrantes con otro tipo de entidades, partidos políticos y administraciones públicas. Por ello, consideramos necesario desarrollar brevemente los conceptos de capital social y de integración política sobre los que trabajamos, aplicados concretamente a la realidad migratoria. 
Esta investigación ${ }^{1}$ parte de un enfoque reticular con la mirada puesta en las redes formales e informales tejidas por la población migrante, lo que nos lleva a adoptar una concepción de capital social próxima a la definición realizada por Bourdieu sobre este término: «acumulación de recursos reales o potenciales que están unidos a la posesión de una red duradera de relaciones más o menos institucionalizadas de reconocimiento mutuo» (Bourdieu, 1986). De este modo, el capital social es fruto de los activos que incorporan los miembros a la estructura reticular en la que se insertan a través de las relaciones tejidas y debido a la posibilidad de acceder a los recursos de dicha red (Lin, 2002). Partiendo del prisma individualista que nos ofrece Bourdieu, donde el capital social es un recurso inalienablemente apropiado por el actor, vemos que su componente de pertenencia a la red social otorga las propiedades comunes de apropiación colectiva a todos sus miembros (Bourdieu, 2001). Es decir, los resultados de la acción de los sujetos se ve determinada por las redes sociales de las que forman parte, y la inclusión en dichas redes permite a sus miembros compartir los recursos disponibles en la misma.

Sin embargo, cuando hablamos de capital social en el campo político es necesario incorporar el marco que establece las pautas de participación y relación, con lo que nos sumamos a la tesis expuesta por Stolle (2000: 5), según la cual la diferencia culturalista y estructuralista de la teoría del capital social no responde a un paradigma diferenciado, sino a los distintos enfoques dirigidos hacia el fenómeno de estudio. La dificultad radica en la ausencia de una microteoría del capital social (Stolle, 2000) que aúne ambas dimensiones explicando la relación causal entre la participación en redes y asociaciones con la conformación de dinámicas de confianza y reciprocidad o, en dirección inversa, que muestre cómo el ámbito de la cultura social suscita lógicas que facilitan la generación de redes y asociaciones. Sin pretender encontrar dicha microteoría, esta definición bebe de ambos enfoques en su construcción y en su formulación del concepto de capital social. Todo capital es un recurso que se invierte para obtener un beneficio, por tanto, el capital social, como recurso relacional, es una «inversión en relaciones sociales con expectativas de retorno en el mercado» (Lin, 2002: 19), y este mercado puede ser de naturaleza diversa según el ámbito de observación: económico, político, laboral o comunitario (Lin, 2002). Este capital se activa por parte de los miembros de una red social o de actores sociales a través de las conexiones que establecen con otros nodos con los que les unen lazos fuertes o débiles, y obtienen ventajas tanto en un plano individual — accediendo a recursos que le son ajenos - como grupal —ampliando la red y, por tanto, los recursos potenciales.

El capital social desempeña un papel protagónico en un doble sentido. Por un lado, generando relaciones entre grupos diversos mediante la potenciación

1. Este artículo forma parte de los resultados de la investigación "Asociacionismo inmigrante en Galicia. Lazos transnacionales y codesarrollo desde una perspectiva de género", dirigida por la profesora Antía Pérez Caramés (Universidade da Coruña) y financiada por Cooperación Galega [Xunta de Galicia] en los años 2014 y 2015. 
de dinámicas de interconexión social a través de redes extrafamiliares (bridging social capital) conformadas por vínculos débiles (Grannovetter, 1973). Por otro lado, fortaleciendo la cohesión interna de los grupos (bonding social capital) a través de estructuras cerradas que ejercen el control social (Coleman, 2001). Las redes sociales serían vistas como el complejo entramado de relaciones entre actores sociales (Requena, 1989). Las redes son las estructuras por las que circula la información, los bienes o los apoyos materiales o emocionales (Requena, 1998).

La participación en entidades de inmigrantes se ha constatado como positiva para la integración en las sociedades de destino (Guarnizo et al., 2003), de la misma forma que la integración social promueve una participación política, lo que se puede concretar en una mayor probabilidad de votar en las elecciones municipales (Tillie, 2004) o en un mayor interés por la política en la sociedad receptora (Morales y Pilati, 2011; Pilati y Morales, 2016).

A ello se suma que dicha participación se orienta hacia intereses individuales y colectivos como migrantes, lo que genera dinámicas identitarias respecto al país de destino y de defensa de derechos. La militancia en organizaciones de inmigrantes está asociada positivamente con una mayor participación en organizaciones proderechos de los inmigrantes frente a organizaciones generalistas de la sociedad civil (Pilati y Morales, 2016).

En esta línea, la participación política se entiende como aquellas actividades llevadas a cabo por personas del común dirigidas a influir, directa o indirectamente, en decisiones tomadas en los diversos niveles del sistema político (De Rooij, 2012, citando a Brady, 1999, y a Verba, 1995). Desde las políticas públicas de integración de inmigrantes en España se estimuló la creación y la consolidación de asociaciones de inmigrantes (Cebolla Boado y López Sala, 2012), porque se consideraba que la existencia de estos actores era un indicador de incorporación exitosa a la sociedad de destino (Cebolla Boado y López Sala, 2012). Los mecanismos elegidos para esta promoción han sido varios, desde el propio apoyo y los incentivos por parte de los técnicos a la creación de asociaciones hasta las subvenciones, pasando finalmente por la creación de órganos o de foros de participación sobre temas de inmigración (con una composición importante de asociaciones de inmigrantes).

La investigación que presentamos aborda la integración política como la participación pública en la sociedad de destino y nos acerca al término integración política definido por González Ferrer y Morales (2006) como «la participación efectiva en el ámbito público por parte de la población de origen inmigrante» (González Ferrer y Morales, 2006: 132), además de incorporar la diversidad participativa y su plasmación asociativa, considerándola un efecto de la apropiación colectiva de los migrantes de fórmulas de transformación y de presencia social.

En este artículo seguimos, de forma aproximada - pero partiendo de metodologías diversas y de resultados de investigación distintos-, los trabajos sobre integración política organizativa de González Ferrer y Morales. Sus investigaciones dividen dicha noción en dos componentes: 
1. Los derechos de acceso a la toma de decisiones a través de la inclusión formal o informal en organismos o en mecanismos de toma de decisiones.

2. La participación efectiva, que sería considerada el conjunto de actividades políticas, el contacto con organizaciones de dicho ámbito en la sociedad de destino y los contactos transnacionales con agentes de su país de origen (González Ferrer y Morales, 2006).

En este caso, intentaremos ver el aprovechamiento, por parte de las asociaciones de inmigrantes, de la estructura de oportunidades y restricciones políticas (Koopmans y Statham, 2000) que brindan los ámbitos local y autonómico de actuación. En definitiva, analizaremos las redes y el capital social de los inmigrantes como factores relevantes en la integración política en los distintos ámbitos.

\section{Metodología}

Este artículo es fruto del proyecto de investigación Asociacionismo migrante en Galicia: Lazos transnacionais e codesenvolvemento desde unha perspectiva de xénero, desarrollado por la Universidade da Coruña durante los años 2014 y 2015 y financiado por la Xunta de Galicia. El estudio combinó la recogida de información cuantitativa con las técnicas de análisis cualitativo. En el mismo se recogen algunos de los principales resultados de dicha investigación.

El punto de partida fue la elaboración de un censo que incorporó todas las asociaciones migrantes implantadas en el territorio gallego e inscritas en los registros provinciales y autonómicos. Este registro contaba inicialmente con 179 asociaciones y posteriormente fue depurado. Se discriminaron a aquellas realmente activas en el tejido asociativo de Galicia frente a las que tenían una presencia meramente formal en él. Finalmente, fueron 89 las organizaciones consideradas activas, lo que indica una tasa de mortalidad del $50 \%$ desde la etapa comprendida entre 2009 (año de partida de las inscripciones registrales) y 2016. Estos registros no miden bien la desaparición de las asociaciones. Inscribirse es fácil, pero casi nadie notifica su defunción.

De las 89 entidades consideradas activas en Galicia, 32 —aproximadamente un 36\% de ellas- accedieron a responder a un cuestionario a través de contacto telefónico sobre tres bloques temáticos: trayectoria migratoria; experiencia participativa e implicación con la comunidad de origen, e historia y fines de la asociación. Esta tasa de respuesta es similar al estudio realizado sobre asociacionismo inmigrante no comunitario en la Comunidad Valenciana, que alcanza un 41\% (Cloquell, 2014: 318). Los resultados del Cuestionario a Asociaciones de Inmigrantes en Galicia nos permiten describir las principales características del tejido asociativo inmigrante en dicha comunidad y evaluar la integración política de las personas foráneas a través de las redes asociativas en función de la presencia política formal e informal, la interacción entre entidades o con instituciones públicas o políticas. 
No obstante, el grueso del proyecto recae en un conjunto de entrevistas semiestructuradas a líderes asociativos migrantes, con la participación de integrantes de 25 organizaciones y, por tanto, con un alcance del $28 \%$ del tejido asociativo activo migrante, a lo que se suman 18 entrevistas semiestructuradas a agentes clave y a expertos vinculados a instituciones autonómicas y a entidades sin ánimo de lucro sobre asociacionismo migrante gallego. A través de dichas entrevistas se buscó la contextualización y la explicación de las evidencias recogidas mediante el Cuestionario a Asociaciones de Inmigrantes en Galicia. Además, se abordó la meta de explorar la evolución del apoyo gubernamental a las políticas específicas de inmigración. Concretamente, se indagó acerca de la relevancia concedida a las asociaciones migrantes como interlocutoras.

\section{El contexto migratorio gallego}

Galicia es un territorio en el que se cruzan procesos migratorios históricos y contemporáneos. En el presente capítulo explotaremos la dinámica migratoria de Galicia como receptora de flujos de retorno de gallegos procedentes de las salidas de la década de 1950 a Latinoamérica y de 1960 a países europeos; a lo que sumaremos la llegada de inmigración extranjera con y sin vínculos históricos con la emigración gallega, y, finalmente, la «nueva emigración gallega», que, a raíz de la actual crisis económica, sale del país a las ciudades globales en busca de mejores perspectivas profesionales.

En Galicia residen 87.000 personas de nacionalidad extranjera, según datos del Padrón Municipal de Habitantes (INE, 2017), lo que significa apenas un $3 \%$ del conjunto poblacional. El norte peninsular fue y es un destino poco atractivo para la llegada de inmigrantes extranjeros. La crisis económica asociada con reemigración y retorno, junto con las concesiones de nacionalidad española - 33.000 entre 2002 y 2014 - , provocaron un descenso de 25.000 extranjeros en el total de empadronados, cifra que llegó a su cénit en el año 2012, con 112.000 inmigrantes. En definitiva, la inmigración extranjera está infrarrepresentada en Galicia en relación con el peso poblacional de la zona en el conjunto del Estado, que equivale a un 6\% (INE, 2017).

La composición del total de extranjeros suele analizarse a través de las nacionalidades principales. La inmigración fronteriza de portugueses representa un $17 \%$ en 2017 , por lo que resulta el colectivo más numeroso. En orden de importancia les sigue el colectivo rumano, que representa un 10\% del total; un $8 \%$ está formado por personas de origen brasileño y marroquí; un $5 \%$ son venezolanos y colombianos; un $4 \%$ cuenta con la nacionalidad italiana (pero una parte importante de dicho porcentaje está formado por argentinos de procedencia que no han residido en Italia) y dominicana, y, finalmente, un 3\% procede de China, Senegal y Argentina. En definitiva, las migraciones laborales y sin vínculos previos con Galicia (Rumanía, Marruecos, Colombia, República Dominicana, China y Senegal) representan un 32\% del total y, junto con la llegada de los vecinos portugueses (y los nacionales brasileños), que aportan 
Gráfico 1. Evolución de las principales nacionalidades de población extranjera empadronada en Galicia (1998-2017)

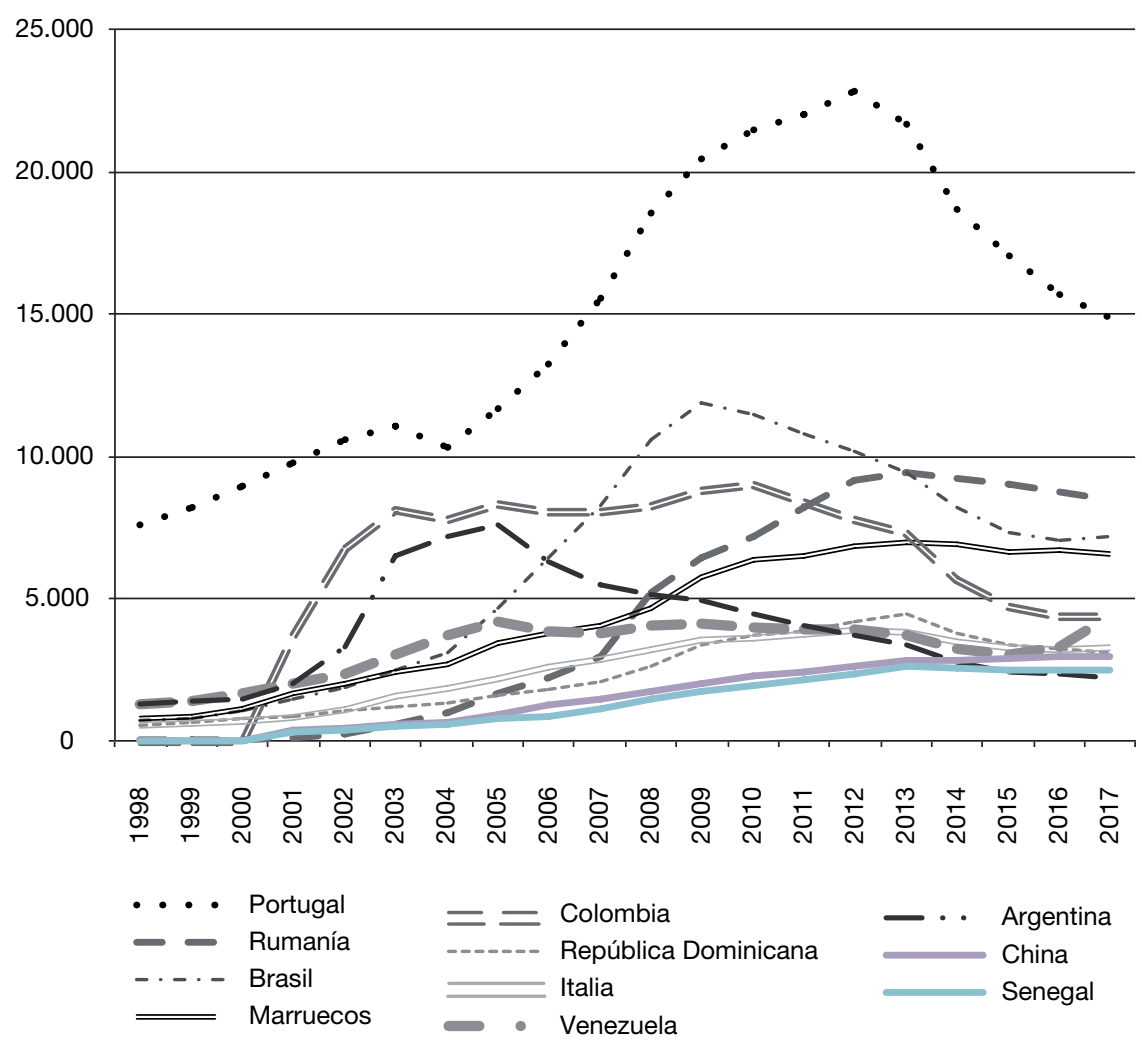

Fuente: elaboración propia a partir de los datos del Padrón Municipal de Habitantes (INE, 1998-2017).

otro $25 \%$, protagonizan la mayor parte del asentamiento migratorio en Galicia en 2017 (Pérez Caramés, 2017).

En grandes líneas, el contexto migratorio gallego, poco atrayente para la inmigración foránea y con un pausado dinamismo económico, es un factor a considerar a la hora de entender que estamos ante un total poblacional de extranjeros que son residentes permanentes, y que sobre todo se trata de una inmigración laboral. A pesar de la baja intensidad, podemos decir que se incentivó la creación de asociaciones de inmigrantes a través de políticas públicas autonómicas de integración, proceso que quedó frenado en el año 2011 por el descenso del gasto social. La crisis, que impactó en gran medida en la población foránea asentada en Galicia - lo que provocó una importante exclusión laboral y social—, se llevó también las ayudas específicas al asociacionismo inmigrante, en definitiva, al principal mecanismo de participación política de este colectivo. 


\section{Los números de la integración política de las asociaciones de inmigrantes en Galicia}

Los datos extraídos del Cuestionario a Asociaciones de Inmigrantes en Galicia, administrado por teléfono a 32 entidades de inmigrantes, nos permiten elaborar, en primer lugar, un pequeño diagnóstico descriptivo de los rasgos estructurales de las entidades de inmigrantes en Galicia. Dicho diagnóstico tiene relevancia académica, pues prácticamente no existen investigaciones que analicen la realidad de este tejido en la zona (Vázquez Silva, 2007 y 2017), como sí sucede con mayor profusión respecto a otras comunidades autónomas. En segundo lugar, nos centraremos en la participación política de dichas organizaciones. Lo haremos a través de la explotación de este cuestionario de variables como el contacto con otras entidades, instituciones públicas y actores sociales y políticos.

\subsection{Breve perfil del tejido asociativo en Galicia}

Para comenzar, debemos señalar que la totalidad de las asociaciones están incorporadas en un registro oficial que puede verse como un indicador de presencia política formal. Concretamente, un $34 \%$ se ha inscrito en un solo registro; un 53\%, en dos registros administrativos, y, finalmente, solo un 13\% ha triplicado este proceso administrativo, teniendo en cuenta que la máxima opción era anotarse en cuatro censos de asociaciones (estatal, autonómico, provincial y local). Un $84,4 \%$ ha optado por dar de alta la entidad en un registro municipal, un $22 \%$ ha realizado este proceso en el ámbito provincial, un $66 \%$ ha dado este paso en el ámbito autonómico y, finalmente, solo un $6,3 \%$ está anotado en un registro estatal. Estos datos nos muestran una fuerte institucionalización, tanto a nivel local como autonómico.

En relación con las principales procedencias de las asociaciones, nos encontramos con que un $50 \%$ de las mismas es de origen latinoamericano, un $28 \%$ está compuesto por inmigrantes de países del África subsahariana, un 13\% muestra una composición mixta de inmigrantes de procedencias diversas, un $6 \%$ corresponde a entidades procedentes del Magreb y, finalmente, un $3 \%$ está formado por organizaciones cuyos miembros tienen nacionalidades de la UE-28. Si tomamos como referencia los datos del Padrón Municipal de Habitantes a fecha 1 de enero de 2016 (Instituto Nacional de Estadística), nos encontramos con que las asociaciones latinoamericanas y africanas de inmigrantes están sobrerrepresentadas, porque su peso se encuentra por encima del $37 \%$ del total de población extranjera para el caso de nacionalidades latinoamericanas y del 13\% del peso de la población del África subsahariana en Galicia. El peso de las asociaciones de la zona del Magreb se corresponde con el $7 \%$ de su total poblacional en Galicia. Finalmente, está infrarrepresentada la población procedente de la UE-28, que supone un $40 \%$ del total de las personas extranjeras empadronadas en Galicia en 2016. Como suele ser habitual, no existe ninguna asociación de población asiática en la muestra realizada, mien- 
Gráfico 2. Porcentajes de población extranjera por áreas de procedencia principales (2016) y de asociaciones de inmigrantes en Galicia por áreas de procedencia principales (2015)

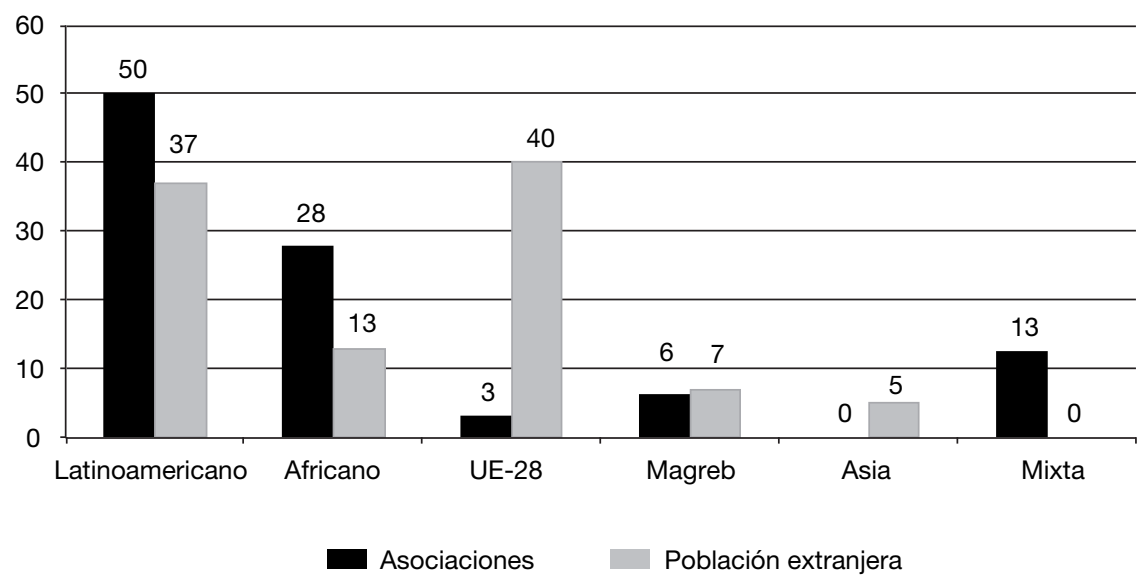

Fuente: elaboración propia a partir del Padrón Municipal de Habitantes a fecha 1 de enero de 2016 (Instituto Nacional de Estadística) y Cuestionario a asociaciones de inmigrantes en Galicia.

tras que su peso poblacional se ubica en torno al 5\% de la población extranjera. Por lo tanto, resulta claro que el lugar de procedencia es una variable que incide en la mayor o menor propensión a crear entidades asociativas de inmigrantes.

La media de personas asociadas a este tipo de entidades en Galicia es de 131, con una desviación típica de 196, en parte porque existen dos entidades con altos niveles de participación migrante. Es complicado obtener datos sobre participación en las asociaciones (Lacomba y Giner, 2013), pero los que disponemos acerca de la Comunidad Valenciana señalan que la mayoría de las entidades se sitúan en una franja entre los 25 y los 100 miembros en general, aunque las asociaciones de marroquíes y senegaleses superan la media de los 200 afiliados (Cloquell, 2014). En el caso concreto de las asociaciones de inmigrantes africanos en Valencia, Navarra y Cataluña, la media de socios es de 153 personas, aunque se percibe diferencia en las medias de estas entidades en las distintas comunidades (Garreta y Llevot, 2013). En líneas generales, y a pesar de la gran dispersión, podemos percibir que el tamaño de su composición es reducido comparativamente, aunque más voluminoso en las agrupaciones de composición africana.

Solo 3 de las 32 asociaciones, es decir, un 9,4\%, cuenta con personal contratado para apoyar el funcionamiento de la entidad en Galicia. Estudios comparativos nos indican que las cifras son mayores para el tejido asociativo inmigrante en la Comunidad Valenciana, que alcanza un 19\% (Cloquell, 2014), y para las asociaciones africanas la media en Cataluña, Valencia y Navarra indica que solo un 5,3\% cuenta con personal contratado, siendo más alta en la Comunidad Valenciana (Garreta y Llevot, 2013). En cuanto a la participación de la masa de socios, de media un $49 \%$ de los inscritos participa de 
forma activa, aunque aquí también tenemos una alta desviación típica para Galicia. Sobre el grado de participación, podemos señalar que el estudio para la Comunidad Valenciana indica ratios más bajas —entre el 10 y el 15\%- y una menor participación activa en las entidades más voluminosas (Cloquell, 2014).

En el caso gallego, la junta directiva está compuesta por una media de 6 personas, con una desviación típica de 2 personas, y un 50\% están lideradas por mujeres. Estas cifras son similares en la investigación de asociaciones de inmigrantes africanas en Cataluña, Navarra y Valencia, donde la media de miembros se sitúa entre los 7 u 8 (Garreta y Llevot, 2013). La cuota media anual que paga cada socio o socia asciende a 28 euros, y en este caso la desviación típica es elevada, puesto que alcanza los 33 euros. La media de ingresos anuales de las entidades en el año 2014 es de poco más de 3.000 euros, pero cuenta asimismo con una fuerte desviación típica de 6.519 euros. Su presupuesto procede principalmente del pago de cuotas - que es el ingreso mayoritario también de las entidades de inmigrantes en Valencia (Cloquell, 2014)—, en menor medida, de donaciones y de campañas de recaudación de fondos, y en algunos casos cuentan con una aportación municipal —un $22 \%$ de las entidades que contestaron, es decir, 6 de 27-. Con financiación autonómica y provincial solo encontramos a una única entidad que dispone de ayuda para su mantenimiento y funcionamiento. Si antes de la crisis podíamos hablar de su dependencia de fondos públicos, quizás en este contexto de austeridad en el gasto social podemos hablar de entidades cuasi autofinanciadas.

En cuanto a la presencia política informal que puede medirse como su integración en alguna plataforma o en algún colectivo no registrado, aquí nos encontramos con que solo un $31,3 \%$ de las entidades pertenece a alguna plataforma colectiva; es decir, 10 de un total de 32 asociaciones. Estas son cifras similares a las de la participación de asociaciones de inmigrantes africanos en Cataluña, Valencia y Navarra en plataformas o foros que alcanza un 22\% (Garreta y Llevot, 2013: 25). Las asociaciones que están vinculadas al Foro Galego de Inmigración, una plataforma que aglutina entidades de inmigrantes, representan un 12,6\% del total; dos organizaciones pertenecen a la Federación de Venezolanos en Galicia, lo que equivale a un peso del 6,2\%; también encontramos a una entidad asociada a la Federación de Asociaciones de Peruanos de Galicia; otra, a la Asociación Nacional de Inmigrantes de Cabo Verde; otra, a la Federación Senegalesa de Galicia, y, finalmente, una de ellas forma parte de una plataforma de tipo religioso de Discípulos de Touba en Galicia. En definitiva, no existe una tendencia a la concentración ni a la agrupación entre entidades en plataformas, lo cual, si cabe, provoca una cierta dispersión de sus actividades y reduce su capacidad de influencia política.

\subsection{El asociacionismo inmigrante en Galicia y su participación política}

La participación en la vida de las asociaciones es lo que se conoce como capital social étnico (Jacobs y Tillie, 2004), y la densidad de la red entre asociaciones étnicas conectadas horizontalmente entre sí se percibe como una mayor 
Tabla 1. Percepción de la importancia de los contactos de las asociaciones de inmigrantes en relación con otros actores sociales y políticos en Galicia (2015)

\begin{tabular}{lcrrrr}
\hline & $\begin{array}{c}\text { Muy } \\
\text { importante }\end{array}$ & $\begin{array}{c}\text { Algo } \\
\text { importante }\end{array}$ & $\begin{array}{c}\text { Poco } \\
\text { importante }\end{array}$ & $\begin{array}{c}\text { Nada } \\
\text { importante }\end{array}$ & Total \\
\hline Contacto con asociaciones de inmigrantes & 84,4 & 9,4 & 6,3 & 0,0 & 100 \\
Contacto con ONG de España 0 Galicia & 71,9 & 21,9 & 3,1 & 3,1 & 100 \\
Contacto con asociaciones u ONG del país de origen & 56,3 & 15,6 & 12,5 & 15,6 & 100 \\
Contacto con instituciones del Gobierno del país de origen & 50,0 & 18,8 & 18,8 & 12,5 & 100 \\
Contacto con los partidos políticos & 18,8 & 21,9 & 28,1 & 31,3 & 100 \\
Contacto con los sindicatos & 21,9 & 15,6 & 25,0 & 37,5 & 100 \\
Contacto con el Ayuntamiento & 59,4 & 21,9 & 9,4 & 9,4 & 100 \\
Contacto con el Gobierno autonómico & 43,8 & 25,0 & 15,6 & 15,6 & 100 \\
Contacto con el Gobierno central & 28,1 & 21,9 & 18,8 & 31,3 & 100 \\
Contacto con las instituciones europeas & 28,1 & 21,9 & 15,6 & 34,4 & 100 \\
\hline
\end{tabular}

Fuente: elaboración propia a partir del cuestionario a asociaciones de inmigrantes en Galicia.

propensión a la participación política (Fennema y Tillie, 1999; Tillie, 2004). La valoración positiva de la interacción interasociativa, y más aún si su conexión horizontal es intensa, puede lograr un aumento de la confianza social, la cooperación voluntaria y reforzar las normas de reciprocidad (Putnam, 2003; Ostrom y Ahn, 2003). En nuestro estudio, esta interacción y este contacto entre organizaciones de inmigrantes y otro tipo de actores sociales y políticos se analizarán como elementos positivos de integración social y política.

Para medir estos contactos, nos quedaremos previamente con la visión de las entidades sobre si dicha relación con otros actores resulta significativa y relevante en relación con sus propios fines. Las entidades de inmigrantes perciben que el contacto con otras asociaciones de inmigrantes y con las ONG de la sociedad de destino es muy importante o algo importante en un 94\% de los casos. Desciende su consideración del grado de transcendencia de tener relación con asociaciones o entidades del tercer sector de su país de origen hasta alcanzar el 72\%, y la conexión con las instituciones del gobierno del país de origen es central para el 69\% de las organizaciones entrevistadas. Su valoración de la importancia de tener contacto con partidos políticos solo resulta significativa para un $41 \%$ de las entidades, y desciende a un $37 \%$ si nos referimos al trato con los sindicatos. Cuando hablamos de instituciones públicas, perciben como más relevantes la comunicación con ayuntamientos en un $81 \%$ de los casos, un $69 \%$ ve significativo el contacto con la Administración autonómica y un 50\% opina lo mismo sobre el Gobierno central y las instituciones europeas. En conclusión, podemos decir que priorizan la conexión con entidades similares a las suyas y con el tercer sector. En un segundo plano aparece la relevancia de las relaciones transnacionales, y, finalmente, tienen una importancia especial los ámbitos municipal y autonómico frente al estatal y europeo. En cambio, quitan valor al contacto con partidos políticos y sindicatos. 
La poca importancia otorgada por estas organizaciones a la interacción con los partidos, medida por una pregunta en la que se valora la relevancia de los actores políticos por parte del tejido asociativo inmigrante, tiene su consecuencia posterior en una falta de vinculación real con los mismos ${ }^{2}$. Ello se infiere de una pregunta acerca de si la entidad está próxima a alguna fuerza política. El contacto continuo con los partidos en general no alcanza a dos de cada diez organizaciones de inmigrantes. El 19\% de los líderes asociativos extranjeros declara tener un contacto habitual con el Partido Socialista Obrero Español, mientras que el 15\% mantiene trato con el Partido Popular o con las Mareas y se reduce respecto al resto de fuerzas políticas, con las que la relación es menor y solo hay contacto para un $9 \%$ de las entidades entrevistadas. Finalmente, solo un $12,5 \%$, es decir, tres de las asociaciones de inmigrantes se consideraban próximas a algún partido político, concretamente, dos entidades manifestaron estar próximas al Partido Socialista y una a Ciudadanos. Por así decirlo, estos datos alejan el fantasma del riesgo de la cooptación política de las entidades de inmigrantes afincadas en Galicia, como puede suceder en otros niveles administrativos como el estatal y en otro momento temporal y político (Veredas Muñoz, 2003).

Retomando el análisis del contacto y la frecuencia entre organizaciones, nos encontramos con que, para las asociaciones analizadas, se producen comunicaciones habituales con asociaciones de inmigrantes en un $47 \%$ de los casos; en cambio, en un $34,4 \%$ de los casos, la relación es constante con las entidades del tercer sector (ver la tabla 2). Las conexiones entre estas organizaciones y las instituciones públicas son frecuentes, especialmente con el Área de Migraciones y con el Departamento de Servicios Sociales del Ayuntamiento. En un 37,5\% de los casos denotan relación directa y constante con dichas instituciones municipales, lo que contrasta con el contacto con las del país de origen, con las que tan solo el $22 \%$ de las organizaciones tiene un trato habitual.

La fortaleza del contacto de las entidades de inmigrantes con las centrales sindicales es variable. Este aspecto se mide a través de una autodeclaración en una pregunta del cuestionario que apela a la percepción del informante que habla en nombre de la organización. El contacto con Comisiones Obreras es constante para un $22 \%$ de las entidades de inmigrantes que señala un trato habitual, frente a un vínculo débil con la Unión General de Trabajadores (UGT) y la Confederación Intersindical Galega (CIG), con quien solo el 6\% de las organizaciones declara tener relación frecuente.

2. La valoración sobre la relevancia en el contexto se obtiene a través de una declaración de la asociación ante la siguiente pregunta cerrada: «¿Podría decirme si, para su asociación, el contacto con $x$ es muy importante, algo, poco o nada importante?». Posteriormente, y después de contestar a la importancia del contacto - habitual, esporádico o ninguna aproximación - con partidos políticos, se pasa a indagar si existió contacto con las distintas fuerzas políticas (Partido Popular, Partido Socialista, con las Mareas — en los que se incluyen a ANOVA, Izquierda Unida o Podemos, así como las candidaturas municipalistas- y con el Bloque Nacionalista Galego). Finalmente, se procede a preguntar directamente si la entidad se siente cercana a algún partido político y se pregunta a cuál. 
Tabla 2. Contacto y frecuencia del mismo mantenido por las asociaciones de inmigrantes en Galicia (2015)

\begin{tabular}{|c|c|c|c|c|}
\hline & $\begin{array}{l}\text { Contacto } \\
\text { habitual }\end{array}$ & $\begin{array}{l}\text { Contacto } \\
\text { esporádico }\end{array}$ & $\begin{array}{l}\text { Ningún } \\
\text { contacto }\end{array}$ & NS/NC \\
\hline Contacto con asociaciones de inmigrantes & 46,9 & 37,5 & 12,5 & 3,1 \\
\hline Contacto con ONG de España o Galicia & 34,4 & 34,4 & 28,1 & 3,1 \\
\hline Contacto con asociaciones o ONG del país de origen & 6,3 & 31,3 & 59,4 & 3,1 \\
\hline Contacto con instituciones del Gobierno del país de origen & 21,9 & 18,8 & 56,3 & 3,1 \\
\hline Contacto con concejales o representantes políticos del PP & 15,6 & 25,0 & 50,0 & 9,4 \\
\hline Contacto con concejales o representantes políticos del PSOE & 18,8 & 25,0 & 46,9 & 9,4 \\
\hline Contacto con concejales o representantes políticos de las Mareas & 15,6 & 18,8 & 56,3 & 9,4 \\
\hline Contacto con concejales o representantes políticos del BNG & 9,4 & 21,9 & 59,4 & 9,4 \\
\hline Contacto con concejales o representantes políticos de otros partidos & 9,4 & 15,6 & 65,6 & 9,4 \\
\hline Contacto con CC. 00. & 21,9 & 6,3 & 68,8 & 3,1 \\
\hline Contacto con UGT & 6,3 & 6,3 & 81,3 & 6,3 \\
\hline Contacto con CIG & 6,3 & 9,4 & 78,1 & 6,3 \\
\hline Contacto con otros sindicatos & 0,0 & 3,1 & 84,4 & 12,5 \\
\hline Contacto con el Área de Migraciones a nivel municipal & 37,5 & 25,0 & 25,0 & 12,5 \\
\hline Contacto con el Área de Servicios Sociales a nivel municipal & 37,5 & 34,4 & 31,9 & 6,3 \\
\hline Contacto con los colegios de su zona & 18,8 & 34,4 & 43,8 & 3,1 \\
\hline Contacto con la policía local & 15,6 & 31,3 & 50,0 & 3,1 \\
\hline Contacto con los centros de salud de su zona & 9,4 & 21,9 & 62,5 & 6,3 \\
\hline Contacto con la Consellería de Política Social (Xunta) & 9,4 & 31,3 & 53,1 & 6,3 \\
\hline Contacto con la Consellería de Emprego (Xunta) & 9,4 & 18,8 & 65,6 & 6,3 \\
\hline Contacto con la Consellería de Educación (Xunta) & 9,4 & 15,6 & 68,8 & 6,3 \\
\hline Contacto con el Ministerio de Empleo y Seguridad Social & 9,4 & 12,5 & 75,0 & 3,1 \\
\hline Contacto con el Ministerio de Educación & 3,1 & 6,3 & 87,5 & 3,1 \\
\hline Contacto con el Ministerio del Interior & 9,4 & 9,4 & 78,1 & 3,1 \\
\hline Contacto con las instituciones europeas & 3,1 & 15,6 & 78,1 & 3,1 \\
\hline
\end{tabular}

Fuente: elaboración propia a partir del Cuestionario a asociaciones de inmigrantes en Galicia.

En definitiva, la mayor intensidad de los contactos se produce con asociaciones de inmigrantes y entidades sin ánimo de lucro en la sociedad de destino, al igual que con el área municipal encargada de la atención a población extranjera o con los servicios sociales municipales. Esto refuerza el carácter local de los contactos de las entidades y también sus relaciones entre iguales — asociaciones de inmigrantes - y semejantes —entidades del tercer sector.

En conclusión, el grado de institucionalización de las asociaciones de inmigrantes es importante en cuanto a su inclusión en registros administrativos. Además, dichas organizaciones mantienen una relación relevante con entidades que trabajan con población extranjera y con sus otras entidades homólogas formadas por población extranjera. También mantienen una relación habitual con la Administración municipal, más concretamente con las áreas especializadas en atención a la población extranjera —áreas de migraciones y servicios sociales. 


\section{Los discursos de asociaciones de inmigrantes y de actores clave sobre la integración política de tejido social migrante}

El aumento de los flujos migratorios en la última década no ha derivado en la articulación de un movimiento asociativo inmigrante cohesionado e influyente en el Estado español. Por contra, las características que lo definen son la fragmentación étnico-nacional (Toral, 2010) y la falta de coalición del movimiento organizado, tanto en la escala autonómica (Masanet Ripoll y Santacreu Fernández, 2010) como en la estatal (Gómez Gil, 2006). En el caso de Galicia, la estructura organizativa conformada por la población inmigrante adolece de la problemática común que acompaña a otras zonas del Estado — singularizada por un asociacionismo débil fruto de una participación escasa de la población migrante, poco cohesionada más allá de vínculos puntuales e instrumentales $y$, por tanto, con una acción y una movilización desestructuradas.

En el asociacionismo migrante de Galicia nos encontramos principalmente con que los objetivos de las entidades están orientados al mantenimiento de su cultura de origen - dimensión étnica e identitaria- y dirigidos al apoyo mutuo entre compatriotas - dimensión asistencialista- (Veredas Muñoz, 2003). Esto último provoca que existan pocas entidades con una composición mixta, puesto que son hegemónicas las que están enfocadas a agrupar a individuos de una misma procedencia. Estas organizaciones distan mucho de querer ejercer como grupo de presión política, y en muchos de los casos se definen como apolíticas, lo que concuerda con el escaso vínculo con los partidos y la mayoría de sindicatos.

De política, no. ¿Qué arreglamos nosotros si somos ochenta? ¿Qué arreglamos haciendo política para allá? No arreglamos nada. (Asociación de inmigrantes procedentes de un país latinoamericano con vínculos migratorios con Galicia 1)

Los líderes comunitarios de estas agrupaciones tienen una tendencia pronunciada a contar con un historial de activismo previo (Veredas Muñoz, 2003) que puede vincularse a cuatro frentes: la acción social, el trabajo sociocultural, la movilización política y, finalmente, la participación de tipo religioso. La participación política en origen tiene un efecto positivo en el compromiso político en destino (Jacobs et al., 2004). Estas experiencias previas en sus países de origen ${ }^{3}$ sirven para entender el funcionamiento y el papel que deben desempeñar estas asociaciones de inmigrantes en las sociedades de destino.

Ya tengo el bagaje. Yo trabajaba con adultos. Los proyectos, no muchos, eran más bien medicina preventiva, porque entonces teníamos un dispensario de nivel básico. Entonces llegaba gente, se le prestaba asistencia médica, odonto-

3. En los discursos analizados, debemos tener en cuenta que solo consideramos la voz de la población «activada» de forma transnacional, tanto en origen como en destino. Es decir, no se contempla el discurso de aquellos que, estando activos en el país de origen, no mantienen esta posición en la sociedad de destino. 
lógica, pero teníamos un proyecto que se llamaba Plan Nueva Sonrisa. Tenemos 92 abuelos. (Asociación de mujeres inmigrantes principalmente de países latinoamericanos)

Trabajé con una [asociación], este..., Club de Madres. [...] nos daban de, este..., de Cáritas. Nos traían víveres, hacíamos vasos de leche y, este... Después hacíamos manualidades, algunas de ellas que sabían habilidades que tengan para tejer, después les hacíamos exposiciones para generarnos, para poder ayudar al que más lo necesitaba de acuerdo con sus necesidades. (Asociación de inmigrantes procedentes de un país latinoamericano 2)

Yo creo que ya desde que... Los procesos políticos, los procesos duros de mi abuelo, ya como que lo vas llevando en la sangre. Entonces no era la típica niña que me gustaran las discotecas, sino que ya trabajé con los salesianos. (...). Yo creo que esa vena ya la tenía marcada. [...] Entonces, a partir de que yo vine aquí, cuando fundamos [nombre de la asociación], somos de los fundadores, hace casi 18 años hemos trabajado en el proceso asociativo. [...] Sí, yo fui activista toda, toda la vida. (Asociación de inmigrantes procedentes de un país latinoamericano 3)

Allí yo mayormente lo que era pertenecía a un ministerio, pero ministerio evangelista. Yo tenía un ministerio evangélico, que aquí se llama evangelista. Pero yo soy evangélico y aquí soy pastor. Yo soy pastor de la Iglesia [...] Bueno, en principio, yo tenía unos treinta o treinta y cuatro, pero con la economía han emigrado. Han tenido que irse a su país anclados, pero seguimos trabajando. [...] Yo trabajaba, porque allí yo trabajaba para una institución, que era capellán federal y yo trabajaba mayormente con el cuerpo de ayudante del presidente y con la comunidad. (Asociación de inmigrantes procedentes de un país latinoamericano 4 )

\subsection{Del peligro de la cooptación politica y de la dependencia de fondos públicos a la escasa incidencia y a la autogestión de las asociaciones de inmigrantes en Galicia}

La concepción de las entidades de inmigrantes como relevantes para las políticas de integración social en España provocó que se pusiesen en marcha líneas de apoyo institucional, tanto por la vía de recursos como por la institucionalización de foros de participación (Cebolla Boado y López Sala, 2012). Durante el período de expansión del gasto social, que afectó también a la política de integración de inmigrantes y que podemos ubicar en la etapa comprendida entre 2004 y 2008, nos encontramos con fondos económicos destinados a estas entidades por las distintas administraciones públicas. Esto provocó el rápido desarrollo de muchas de ellas y la fuerte dependencia de fondos públicos para poder llevar a cabo sus tareas fundamentales. El descenso de gasto social por parte de las administraciones que tuvo lugar desde el año 2009 en adelante provocó una fuerte reducción de las políticas sectoriales que afectó en gran parte a las medidas de integración de la pobla- 
ción extranjera y, como efecto colateral, al corte de apoyo económico a las entidades de inmigrantes.

Yo creo que los problemas que tienen es que, antes de la crisis económica, evidentemente, casi toda asociación de inmigrantes pues tenía su subvención, 3.000 euros, 6.000 euros, que les permitían más o menos mantenerse. Llegó la crisis económica. Quizás al colectivo que más afectó, de toda Galicia, fue al colectivo de las asociaciones de inmigrantes, porque perdieron cualquier tipo de subvención pública y eso hizo que, bueno, que se encontraran en una situación muy complicada, en el sentido de que ya no tenían recursos para mantener ese local o para mantener ciertas actividades... De repente, también se encontraban que sus asociados, o muchos de ellos, acababan en el paro, tenían más problemas y demandaban a la asociación esas soluciones, y esa asociación no estaba creada para ese fin. (Experto en inmigración gallega 1)

Los resultados de nuestra investigación nos muestran que, pese a la priorización de las relaciones interorganizativas dentro del colectivo migrante, son pocas las organizaciones en Galicia que desarrollan proyectos de manera conjunta, incluso que se organizan de forma puntual para llevar a cabo alguna acción o algún servicio. Cuando esto último ocurre, es fruto de relaciones de carácter personal, generalmente asociadas a la pertenencia étnica, que establecen nexos por donde fluye la información y se generan vías de cohesión.

Sí, sí, sobre todo [trabajamos con] unas asociaciones peruanas. Ella nació en Perú y, bueno, tiene muchas relaciones y sobre todo más que nada a la hora de poder trabajar juntos, ¿no? Cuando se hace un proyecto y ese proyecto puede ir en común, no solamente con Latinoamérica, con la asociación Aida, Asociación de Africanas, todo aquello que sea sumar, nosotras de manera rauda y veloz estamos abiertas para todo tipo de actividad y todo tipo de desarrollo con la mayoría de las asociaciones. (Asociación de inmigrantes procedentes de un país latinoamericano 5)

La ausencia de un frente común que aglutine a las organizaciones de inmigrantes en el espacio asociativo supone un límite para la integración de sus miembros (Tillie, 2004) y para la inclusión de sus temas de interés en la agenda política, lo que lleva a que ese espacio de integración no esté conformado por la lucha unificada del colectivo inmigrante organizado y, en su lugar, sea suplido por las relaciones bilaterales de dichas organizaciones con un tercer sector social autóctono orientado al apoyo y a la atención hacia la inmigración. Por tanto, son estas organizaciones las que establecen las líneas de la acción política dirigidas a los inmigrantes, con lo que actúan como asesoras, informantes y representantes.

No, si nosotros tenemos relaciones con otras ONG, como Ecos do Sur, trabajamos más, y también Foro Emigrante Gallego [Foro Galego da Emigración], porque ellos también están aquí con los inmigrantes. Nosotros somos inmigrantes y tenemos que colaborar con ellos. Ecos do Sur también hace mucho 
por nosotros, son casi como asesores. Muchísimas cosas, las informaciones, las solicitudes y todo eso, y también Equus Zebra, al principio cuando empezamos. (Asociación de inmigrantes procedentes de un país africano)

Sin embargo, es importante señalar que, aunque la densidad asociativa supone un factor relevante para la integración y la reivindicación política, esta necesita de una vía directa de diálogo con las instituciones para plasmar las acciones en el desarrollo de una agenda política concreta. Las redes tejidas con las administraciones públicas son determinantes para hablar de la voluntad de incidencia y participación, incluso más allá de las propias redes intraorganizativas de la población inmigrante (Bolíbar Planas, 2013). Es decir, lograr el paso de una integración de la población inmigrante a una integración de la movilización social inmigrante — - reconociéndola, por tanto, como un actor con pleno derecho en la acción política - requiere de la participación en la vida institucional, más allá de las áreas especializadas de atención a la población extranjera.

[...] porque yo con la Diputación tengo más o menos buena relación. Y, para ser sincera, son los únicos que nos han ayudado a nosotros, que nos han apoyado en los proyectos. Tardan un poco, pero nos han apoyado. Bueno, puedo decir que la Xunta nos ha apoyado, nos apoyó, pero bueno, ahora es diferente. (Asociación de mujeres inmigrantes principalmente de países latinoamericanos)

\subsection{Lo personal (y lo local) es politico: lazos débiles y contactos políticos efimeros entre asociaciones de inmigrantes y actores sociales y politicos municipales}

Como veíamos en el apartado anterior, Galicia cuenta con una realidad asociativa inmigrante cuya participación en la vida institucional se focaliza en el ámbito local. El proceso de acercamiento a dichas instituciones es fruto de dos dinámicas complementarias. Por un lado, la cercanía personal de miembros de la organización con participantes en las instituciones públicas. Y, por otro, un reclutamiento por parte de los partidos políticos de los líderes asociativos locales. Incorporación con éxito limitado, debido al reducido contacto que denotan las organizaciones con los partidos políticos.

Sí, por ejemplo, a mí, como presidenta, me llamó Ciudadanos por medio de una chica. Entonces le dije que lo iba a pensar... Entonces busqué información..., y dije "no». Y luego a los días me llama Compromiso $(\mathrm{CxG})$. Bueno..., bueno, de Compromiso, yo conozco, aparte que me enfocó de tal manera que... Yo, en mi país, tiro de izquierda, y bueno..., participé. Estaba de octava. (Asociación de inmigrantes procedentes de un país latinoamericano 6)

Estuvimos con algunos partidos de izquierda que quisieron llevar feministas. Uno de ellos fue el PSOE. Tuvimos una compañera colombiana de la asociación, salió de la asociación y luego perteneció a la Plataforma de Afectados por la Hipoteca. Fue de número dos y llegó a concejal aquí en Fene. (Asociación de inmigrantes procedentes de un país latinoamericano 3) 
Esta adscripción partidista de activistas migrantes no siempre viene acompañada de la admisión de sus demandas, pues, salvo excepciones puntuales, se trata de procesos que buscan la generación de listas electorales diversas, sin que ello se traduzca en asunción de puestos de responsabilidad por parte de dicha población. En parte, ello es debido a la debilidad del movimiento asociativo migrante señalado anteriormente.

Lo único que, por ejemplo, no tanto en Galicia, porque en Galicia realmente, como no han tenido peso, no se les ha manipulado. En otras comunidades autónomas en, de España, se les ha, aprovechando su debilidad... Más que desmovilizarlos, lo que hicieron fue... manipularlos. Aprovechar su asociacionismo para manipularlos [...]. O sea, el tejido social de inmigrantes es muy pequeño y no tiene fortaleza, porque no hay dinero. (Experto en inmigración gallega 2)

El establecimiento de un fenómeno de vinculación institucional por afinidad genera lógicas de integración desigual, tanto desde el punto de vista asociativo como temporal, pues la institucionalización de unas asociaciones u otras es fruto de la confluencia de ideas o de relaciones personales entre miembros de uno u otro colectivo. Y, a la par, dichas relaciones duran lo que lo hace el vínculo entre personas, por lo que un cambio político o una modificación en el equipo directivo de alguna organización puede suponer la ruptura del trabajo conjunto. Todo ello genera un reconocimiento dispar de las organizaciones por parte de la Administración pública en función de sus experiencias organizativas y de la red política tejida.

Mira, este..., el Ayuntamiento bien. También tengo que decir que [se refiere a la entidad] tiene un proceso. No es lo mismo decir «yo soy de [menciona la asociación] o yo soy tal» que decir «yo soy de una asociación de mujeres». Eso también hay que decirlo. No porque [se refiere a la propia entidad] sea más que nadie, lo que pasa es que los años de proceso han propiciado que la entidad tenga la fuerza que tiene. (Asociación de inmigrantes procedentes de un país latinoamericano 3)

Además, la incorporación de los líderes asociativos a la participación institucional puede suponer un freno en la posible actividad movilizada del colectivo. Lo que en la práctica sea, en palabras de Toral (2010), una «integración por exclusión", es decir, la participación en consejos y mesas de diálogo, promovida como estrategia integradora (Cebolla Boado y López Sala, 2012), se torne en una dinámica de desmovilización del colectivo (Della Porta y Diani, 2006). Debido a ello, la presencia en espacios formales de decisión no tiene por qué suponer, en contrapartida, una acción de incidencia política. El grado de integración de la sociedad civil organizada en las estructuras participativas es promovido o limitado institucionalmente mediante el contexto político que facilita o dificulta la politización (Cinalli y Giugni, 2014). De este modo, el papel desempeñado por las instituciones públicas en la modificación de la «estructura de oportunidades políticas» (Ireland, 1994; Koopmans y Statham, 
2000) que permite a las organizaciones inmigrantes incorporarse a la masa civil organizada es de vital importancia. En este sentido, el asociacionismo inmigrante gallego percibe un desinterés en instaurar dinámicas integradoras por parte de la Administración autonómica, señalando que las políticas de integración de la población inmigrante se reducen a un ejercicio de provisión de servicios, subvenciones o apoyos económicos puntuales que no suponen una inclusión real del colectivo y que, a la par, pueden promover dinámicas de cooptación política (Veredas Muñoz, 2003; Lacomba, 2016), lo que limita una participación política formal.

Yo creo que hay relaciones clientelistas o clientelares, pero no en el sentido estricto, sino en el sentido de que no hay realmente un trabajo de participación ciudadana, sino de compra y venta de servicios, es decir, las administraciones públicas ofrecen unos servicios, vamos, entre comillas, «subvenciones», cursos y demás, a los que optan diferentes colectivos [...] Es una suma de cosas (...). Evidentemente, salen interlocutores. Luego, la legitimidad de esos interlocutores será otra cosa, pero si se fomenta el diálogo y la participación, salen interlocutores. Si no se hace, no salen, no hay interlocutor posible. Luego, si hay políticas de fomento de la participación y de la institucionalización de esa participación..., se crean organizaciones, o sea hay ejemplos en España. Con una política de integración, en la que se han fomentado las asociaciones de inmigrantes, se ha constituido un tejido. Aquí en Galicia ha sido mínimo lo que se ha hecho en ese sentido. (Experto en inmigración gallega 2)

Ello contrasta con la aparición de nuevas lógicas de participación lideradas por algunos ayuntamientos «del cambio». Grupos municipales surgidos al calor de las movilizaciones del último lustro, que perciben al migrante como un actor clave en la política local y, por tanto, le hacen partícipe de ello abriéndole nuevos espacios de relación con la Administración ajenos a la estructura consultiva y formal de épocas anteriores. Procesos que se están iniciando y que tardarán un tiempo en demostrar si son capaces de dar respuesta a las necesidades del colectivo inmigrante y a las organizaciones que lo representan, pero que, tal como veíamos anteriormente, no se han plasmado en una gran vinculación con partidos políticos como las Mareas.

Se va a notar muchísimo estos cuatro años pasados, estos cuatro años que vinieron, porque ya se notaron esos cuatro años... de poder tener incluso un diálogo. El sábado que tuvimos nuestra conversación con gentes del Ayuntamiento, los concejales que van a estar al frente sobre inmigración, pues ya es bastante. Ya nos empezarán diciendo: «No hay para nosotros, no va a haber para vosotros». Fue el primer diálogo de la concejala. Eso también va a marcar, yo creo que eso marca muchísimo. Y cada vez que se despierta esa conciencia social que se esta viendo ahora, eso esta bien. Y ahí tenemos que estar aportando nuestro granito de arena nosotros. (Asociación de inmigrantes procedentes de un país latinoamericano 3)

En el ámbito de las prácticas transnacionales, cabe destacar que los proyectos de codesarrollo han sido contados, y solo han participado en ellos las 
asociaciones de inmigrantes cuando el actor político central ha sido la Administración pública autonómica. La complejidad técnica y el conocimiento de la Administración hace que solo entidades del tercer sector, como son las asociaciones Ecodesarrollo Gaia y Ecos do Sur, en la ciudad de A Coruña, hayan puesto en marcha este tipo de proyectos de desarrollo. En el plano local, prácticamente todas las iniciativas se conducen a través del Fondo Galego de Cooperación e Solidariedade, que funciona con aportaciones municipales para posteriormente desarrollar principalmente actividades de sensibilización en las cuales las entidades de inmigrantes tienen un papel marginal. Aunque las referencias a la potencialidad de las asociaciones de inmigrantes como agentes de desarrollo forman parte de la «literatura administrativa» vigente en temas de cooperación en la Comunidad Autónoma de Galicia, no pasan de ser palabras con escasa concreción política.

Nada. O sea, no hay absolutamente nada ni va a haberlo en este plan director. ¿Por qué? Porque no hay recursos, porque no hay el tejido... O sea, el tejido social de inmigrantes es muy pequeño y no tiene fortaleza, porque no hay dinero y porque... Y lo pusieron porque... Había que ponerlo a nivel de... Porque el codesarrollo sí que está dentro de la línea del PP en cooperación. Lo poco que el PP tiene en cooperación, tiene incluido el codesarrollo. De eso se encargó Blasco de meterlo en Madrid y en... Porque, al final, fue Blasco uno de los promotores. (Experto en inmigración gallega 2)

Lo que pasa que suelen ser. Yo la experiencia que tengo con algunas de las que se aprobaron proyectos es que suelen ser asociaciones bastante débiles y con problemas a la hora de justificar, entonces... Entonces el problema está en que si... Si tienen que devolver dinero, como le pasó a una, que no sé ya si... Que prefiero no saber si le devolvió o no devolvió, pero hubo que hacer un reintegro. Entonces, pides una serie de criterios y son demasiado débiles o no están acostumbradas a la mecánica de tener que justificar y tener las... (Experto en inmigración gallega 3)

Por tanto, a modo de resumen, podemos denotar que el asociacionismo inmigrante gallego se caracteriza por una focalización de la actividad en el ámbito local, con estructura relacional débil a nivel intraorganizativo; así como por una escasez de vínculos, de carácter instrumental, con organizaciones de la sociedad civil nativa, lo que contrasta con una relación de mayor intensidad con la Administración local. En definitiva, densidad relacional horizontal débil y dispersa frente a una estructura de vínculos verticales fuerte y concentrada. Rasgos que favorecen la cooptación de los movimientos asociativos (Cinalli, 2007) y limitan su acción política.

\section{Epílogo: ¿De lo global a la estructura local de oportunidades?}

Siguiendo el esquema de González Ferrer y Morales (2006), la participación política del tejido asociativo inmigrante en Galicia se relaciona con el dere- 
cho de acceso a la toma de decisiones, pudiendo afirmar que es escaso y que solo destaca el libre ejercicio del derecho de asociación y, por lo tanto, de inclusión de su entidad en los registros administrativos. Las vías de participación en foros consultivos para estas entidades en el ámbito local y autonómico desaparecieron en un contexto de oportunidades reducido para los temas migratorios. Esta salida de la agenda política está provocada por un cambio de ciclo migratorio que provoca la «emigración de jóvenes gallegos al extranjero" a causa de la crisis económica y del menor gasto social, a la par que se produce una reducción de las estructuras organizativas especializadas en el trabajo con población extranjera. En relación con la participación política efectiva de estas entidades podemos ver cómo los contactos con organizaciones políticas y sociales existen, aunque no son densos, al igual que merman en el plano transnacional, porque en Galicia la apuesta por el codesarrollo que implicaba a las entidades de inmigrantes no llegó a estar en la agenda institucional autonómica.

La investigación que aquí presentamos muestra el enraizamiento del asociacionismo inmigrante gallego en el ámbito local. Las relaciones tejidas con instituciones del tercer sector, que son percibidas como aliadas estratégicas de corte instrumental, se enmarcan en dinámicas de ayuda y atención a la inmigración centradas en el territorio autonómico, mientras que los vínculos entre organizaciones de migrantes también se circunscriben a dicho ámbito e, incluso, cobran fuerza cuando llegamos al nivel municipal. Ello apunta a que las organizaciones de migrantes establecen dinámicas orientadas a satisfacer sus necesidades en las sociedades de destino, relaciones, por tanto, de carácter instrumental. Bien sean demandas de servicios, plasmadas en su relación con las áreas municipales de atención a la población extranjera, bien sea mediante actividades de encuentro y de mantenimiento lingüístico y cultural (Vázquez Silva, 2017) entre colectivos étnicos, lo que se refleja en una composición monoétnica de las organizaciones.

Cuando las entidades de inmigrantes no pueden satisfacer dichas demandas, debido a una reducción en las subvenciones o al cambio de necesidades del colectivo al que representan, su rol se ve limitado como agentes políticos, lo que refuerza el papel instrumental de las mismas. En definitiva, es importante el ciclo migratorio en relación con las peticiones de las asociaciones (Zapata-Barrero, 2004: 148-150), puesto que a mayor antigüedad migratoria se produciría una creciente demanda política. En el caso gallego, ello se vería modificado por la crisis económica y por la respuesta de austeridad en el gasto social, lo que hace que las entidades vuelvan a centrarse en priorizar la satisfacción de necesidades primarias económicas y sociales (Vázquez Silva, 2017).

Por contra, las relaciones con organizaciones o instituciones estatales, federaciones o supraasociaciones de migrantes y asociaciones de compatriotas en origen son muy reducidas y se focalizan en un número escaso de contactos y de organizaciones que las llevan a cabo, las cuales se caracterizan por dinámicas de intercambio personal, y no por lógicas de incidencia política en destino. La ausencia de orientación hacia la realidad en destino, junto a las dificultades que posee el tejido asociativo migrante, por su condición débil y fraccionada, 
ha limitado las opciones de emprender acciones de codesarrollo. La incapacidad técnica de las asociaciones, que en la mayoría de los casos no cuentan con personal de apoyo, se ha sumado a una falta de liderazgo por parte de la Administración autonómica, lo que ha limitado la relación entre la Administración y las asociaciones al campo municipal, especialmente en lo que se refiere a la provisión de servicios a la población migrante y el intercambio cultural.

La importancia del ámbito local como el espacio donde las asociaciones de inmigrantes desarrollan su actividad muestra una lección importante: la necesidad de contar con el contexto sociopolítico municipal para abordar la participación asociativa. De este modo, la «estructura de oportunidades políticas» que determina la posibilidad de integración del asociacionismo migrante se inserta esencialmente en el municipalismo, lo que nos permite apuntar que un cambio en la lógica de funcionamiento institucional que reconozca al migrante como actor con pleno derecho en la acción política, debido, por ejemplo, a la participación de nuevos actores, puede traducirse en una ampliación de la ventana de oportunidad política y, por tanto, en una incorporación efectiva de las asociaciones estudiadas en la política formal.

\section{Referencias bibliográficas}

Bolíbar Planas, Mireia (2013). «Redes asociativas, inmigración y participación: Una aproximación empírica a los vínculos entre capital social y participación asociativa». REDES: Revista Hispana para el Análisis de Redes Sociales, 24(1), 114-136.

BOURDIEU, Pierre (1986). «The forms of capital». En: RiCHARDSON, John G. (ed.). Handbook of theory and research for the sociology of education. Nueva York: Greenwood Press. <https://doi.org/10.2307/2069964>

- (2001). «El capital social: Apuntes provisionales». En: Herreros, Francisco y FrancisCo, Andrés de (eds.). Capital social, Zona Abierta. Madrid: Fundación Pablo Iglesias.

Cebolla BoAdo, Héctor y López Sala, Ana María (2012). «A Top-Down model of transnational immigrant associationism: Migrant organizations in the definition of development and integration policies in Madrid». Princeton University, Woodrow Wilson School of Public and International Affairs, Center for Migration and Development. Working Papers, 1408.

CinALLI, Manlio (2007). "The impact of "relational structures" upon collective action: A comparison of unemployment and asylum in New Labour Britain». Cevipof, Working Paper, 23.

Cinalli, Manlio y GiUgni, Marco (2014). "The Impact of Political Opportunity Structures on the Politicization of Civil Society Organizations in the Field of Unemployment and Precarity». En: BAGLioni, Simone y GiUgni, Marco (eds.). Civil Society Organizations, Unemployment, and Precarity in Europe: Between Service and Policy. Houndmills: Palgrave. $<$ https://doi.org/10.1057/9780230391437.0010>

CloQUell LoZANO, Alexis (2014). «Morfología del movimiento asociativo inmigrante no comunitario: Un estudio empírico al caso de la Comunidad Valenciana». Migraciones, 36, 307-341.

<https://doi.org/10.14422/mig.i36.y2014.003> 
COlEMAN, James (2001). «Social capital in the creation of human capital». En: DASGUPTA, Partha y SERAGELDIN, Ismail (eds.). Social capital: A multifaceted perspective. Washington: World Bank Publications. <https://doi.org/10.1016/b978-0-7506-7222-1.50005-2>

Della PorTa, Donatella y DiAni, Mario (2006). Social Movements: An Introduction. Oxford: Blackwell.

De Roolj, Eline A. (2012). «Patterns of Immigrant Political Participation: Explaining Differences in Types of Political Participation between Immigrants and the Majority Population in Western Europe». European Sociological Review, 28(4), 455-481. $<$ https://doi.org/10.1093/esr/jcr010>

Fennema, Meindert y Tillie, Jean (1999). «Political participation and political trust in Amsterdam: Civic communities and ethnic networks». Journal of Ethnic and Migration Studies, 25(4), 703-726. <https://doi.org/10.1080/1369183x.1999.9976711>

Garreta BochacA, Jordi; Llevot Calvet, Núria (2013). «Las asociaciones de inmigrantes africanos: Organizacion, proyección y actuaciones». Revista Internacional de Sociología, 71(1), 15-38. <https://doi.org/10.3989/ris.2012.09.01>

GÓmEZ GiL, Carlos (2006). «Inmigración y asociacionismo: El caso de Alicante». Observatorio de la Inmigración en Alicante (Universitat d'Alacant). Documento de trabajo.

González Ferrer, Amparo y Morales Diez de Ulzurrun, Laura (2006). «Las asociaciones de inmigrantes en Madrid: Una nota de investigación sobre su grado de integración política». Revista Española del Tercer Sector, 4, 129-173.

Guarnizo, Luis Eduardo; Portes, Alejandro y Haller, Willian J. (2003). «Assimilation and Transnationalism: Determinants of Transnational Political Action among Contemporary Immigrants». American Journal of Sociology, 108(6), 1211-1248. <https://doi.org/10.1086/375195>

GranovetTer, Mark (1973). «The Strength of Weak Ties». American Journal of Sociology, 78(6), 1360-1380.

INE (1998-2017). Padrón Municipal de Habitantes [en línea]. <http://www.ine.es/ dynt3/inebase/es/index.htm?type=pcaxis \&path=/t20/e245/p04/provi/\&file=pca xis\&dh=0\&capsel $=0>$

INE (2017). Padrón Municipal de Habitantes [en línea]. <http://www.ine.es/dyngs/ INEbase/es/categoria.htm?c=Estadistica_P\&cid=1254734710984>

IRELAND, Patrick (1994). The policy challenge of Ethnic Diversity: Immigrant Politics in France and Switzerland. Cambridge: Harvard University Press.

JACOBS, Dirk y Tillie, Jean (2004). «Introduction: Social capital and political integration of migrants». Journal of Ethnic and Migration Studies, 30(3), 419-427. <https://doi.org/10.1080/13691830410001682016>

JaCobS, Dirk; PhaleT, Karen y Swyngedouw, Marc (2004). "Associational membership and political involvement among ethnic minority groups in Brussels». Journal of Ethnic and Migration Studies, 30(3), 543-559. <https://doi.org/10.1080/13691830410001682089>

Koopmans, Ruud y Statham, Paul (2000). "Migration and ethnic relations as a field of political contention: An opportunity structure approach». En: KoOPMANS, Ruud y STATHAM, Paul (eds.). Challenging Immigration and Ethnic Relations Politics. Oxford: Oxford University Press. 
LACOMBA, Joan (2016). «Asociaciones de inmigrantes en la encrucijada: Acción transnacional y riesgos de cooptación». REMHU: Rev. Interdiscip. Mobil. Hum., Brasília, XXIV(47), 27-44.

LACOMBa VÁZqueZ, Joan; Giner MONFORT, Jordi (2013). «La participación en asociaciones de los inmigrantes africanos: Vías de interpretación del distanciamiento asociativo». Revista Internacional de Sociología, 71(1), 67-89. <https://doi.org/10.3989/ris.2012.09.10>

Lin, Nan (2002). Social Capital: A Theory of Social Structure and Action. Cambridge: Cambridge University Press.

Masanet Ripoll, Erika y SANTACreu Fernández, Óscar (2010). «El movimiento asociativo inmigrante en la comunidad valenciana y sus repercusiones en la producción de capital social». Migraciones, 27, 49-81.

MORALES, Laura (2011). «Conceptualizing and measuring migrants' political inclusion». En: MORALES, Laura y GIUGNI, Marco (eds.). Social capital, political participation and migration in Europe: Making Multicultural Democracy Work? Hampshire (UK): Palgrave Macmillan.

Morales, Laura y Pilati, Laura (2011). «The Role of Social Capital in Migrants' Engagement in Local Politics in European Cities». En: MORALES, Laura y GIUGNI, Marco (eds.). Social capital, political participation and migration in Europe: Making Multicultural Democracy Work? Hampshire (UK): Palgrave Macmillan.

Ostrom, Elinor y Ahn, Toh-Kyeong. (2003). «Una perspectiva del capital social desde las ciencias sociales: capital social y acción colectiva». Revista mexicana de sociología, 65(1), 155-233.

PÉREZ CARAmÉs, Antía (2017). «Contexto sociodemográfico da inmigración en Galicia (1996-2016): Unha perspectiva de xénero sobre os cambios recentes no panorama migratorio». En: Rodríguez SALGAdo, Laura; VÁZQUEZ SilVA, Iria (coord.). Muller inmigrante, lingua e sociedade. Vigo: Galaxia, p. 31-60.

Pilati, Katia y Morales, Laura (2016): «Ethnic and immigrant politics vs. mainstream politics: The role of ethnic organizations in shaping the political participation of immigrant-origin individuals in Europe». Ethnic and Racial Studies, 39(15), 2796-2817. <https://doi.org/10.1080/01419870.2016.1181270>

PUTNAM, Robert D. (2003). El declive del capital social: un estudio internacional sobre las sociedades y el sentido comunitario. Madrid: Círculo de Lectores.

REQUENA SANTOS, Félix (1989). «El concepto de red social». Revista Española de Investigaciones Sociológicas, 48, 137-152.

- (1998). "Género, redes de amistad y rendimiento académico». Papers, 56, 233-242.

STOLLe, Dietlind (2000). «Social Capital - A New Research Agenda?: Toward an Attitudinal Approach». ECPR Workshop, 13. Copenhague. Recuperado de <https:// ecpr.eu/Filestore/PaperProposal/b0812d49-9e47-4d19-a0ff-62230487d94f.pdf>.

TilliE, Jean (2004). «Social capital of organizations and their members: Explaining the political integration of immigrants in Amsterdam». Journal of Ethnic and Migration Studies, 30(3), 529-541. <https://doi.org/10.1080/13691830410001682070>

TORAL, Guillermo (2010). «Las asociaciones de inmigrantes como sociedad civil: Un análisis tridimensional». Revista Española de Investigaciones Sociológicas, 132(1), 105-130.

VÁZQUez Silva, Iria (2007). "O incipiente mapa asociativo inmigrante en Galicia». En: VeIRA VeIRA, José Luis (coord.). La sociología y los nuevos retos = A socioloxía 
e os novos retos: IV Congreso Astur-Galaico de Socioloxía. A Coruña: Servizo de Publicacións da Universidade da Coruña.

- (2017). «Inmigración, asociacionismo e ONG: Dificultades, urxencias e desafíos». En: Rodríguez Salgado, Laura y VázQuez Silva, Iria (coord.). Muller inmigrante, lingua e sociedade. Vigo: Galaxia, p. 91-122.

VEREDAS MuÑOZ, Sonia (2003). «Las asociaciones de inmigrantes en España». Revista Internacional de Sociología, 36, 207-225.

ZAPATA-BARRERO, Ricard (2004). Inmigración, innovación política y cultura de acomodación en España. Barcelona: CIDOB. 
